\title{
Bosonic Helium droplets with cationic impurities: onset of electrostriction and snowball effects from quantum calculations
}

\author{
E. Coccia, ${ }^{1}$ E. Bodo, ${ }^{1}$ F. Marinetti, ${ }^{1}$ F. A. Gianturco*, ${ }^{1}$ \\ E. Yildrim, ${ }^{2}$ M. Yurtsever, ${ }^{2}$ and E. Yurtsever ${ }^{3}$ \\ ${ }^{1}$ Department of Chemistry, University of Rome "La Sapienza” and CNISM, \\ Piazzale A. Moro 5, 00185 Rome, Italy \\ ${ }^{2}$ Istanbul Technical University, Chemistry Department, 80626 Maslak, Istanbul, Turkey \\ ${ }^{3}$ Chem. Dep., Koç University Istanbul 34450 Turkey
}

\begin{abstract}
Variational MonteCarlo and Diffusion MonteCarlo calculations have been carried out for cations like $\mathrm{Li}^{+}$, $\mathrm{Na}^{+}$and $\mathrm{K}^{+}$as dopants of small helium clusters over a range of cluster sizes up to about 12 solvent atoms. The interaction has been modelled through a sum-of-potential picture that disregards higher order effects beyond atom-atom and atom-ion contributions. The latter were obtained from highly correlated ab-initio calculations over a broad range of interatomic distances.

This study focuses on two of the most striking features of the microsolvation in a quantum solvent of a cationic dopant: electrostriction and snowball effects. They are here discussed in detail and in relation with the nanoscopic properties of the interaction forces at play within a fully quantum picture of the clusters features.

PACS numbers: $34.20 .-b, 34.30 .+\mathrm{h}$
\end{abstract}

* corresponding author. e-mail: fa.gianturco@ caspur.it fax: +39-06-49913305 


\section{INTRODUCTION}

Our knowledge and understanding of He droplets has witnessed a tremendous growth in recent years, a fact which has contributed, to a large extent, to keep the physics and the chemical physics of liquid helium, and of quantum fluids in general, a lively research field attracting the attention of researchers from many-body theory, molecular and atomic spectroscopy and quantum molecular methods [1, 2, 3, 4, 5, 6]. From the standpoint of picking-up possible impurities, the He droplets are able to do so with any species with which they collide [4] and the latter can then reside either in the inside of the droplet or at its surface depending on the strength of the helium-dopant interaction with respect to the He-He one (e.g. see refs. [7, 8]).

One question about ${ }^{4} \mathrm{He}$ clusters frequently discussed in the literature is the possible existence of magic numbers which are associated with particular configurations that are energetically more stable than neighboring ones and contain a specific number of adatoms [9]. The theoretical estimates for He droplets, in fact, suggested that in the pure aggregates well defined structures are not expected [10] and even recent calculations and experiments [9] connected the presence of such "magic numbers" observed in ${ }^{4} \mathrm{He}$ clusters of small size to surface excitation processes rather than to special stability effects.

The situation, however, is drastically modified when a cationic impurity is being picked up by the droplet: the charged dopant is expected to modify the local environment of the liquid and to give rise to a region of increased density and of electrostriction effects which can cause regular structures to arise around the impurity (snowball effect) [11, 12]. Earlier theoretical studies using "shadow" wavefunctions [13] and modelling large clusters with 60-70 He atoms, have predicted the formation of an easily identifiable first solvation shell [14] where the helium density is well above the critical freezing density of bulk helium so that the first shell can be characterized by a solid-like structure and order parameter [15]. The snowball structure and composition, however, in contrast to the simple snowball model, seem to depend on the nature of the central ion. For the three alkali ions that we are going to discuss in the present work, Galli et al. [14] had initially reported a snowball of 8 atoms for $\mathrm{Li}^{+}$, of 10 for $\mathrm{Na}^{+}$and 12 for $\mathrm{K}^{+}$. A more recent study by the same group [15] has improved their result to 12 for $\mathrm{Na}^{+}$and 15 for $\mathrm{K}^{+}$. A Path Integral Monte Carlo calculation has also been performed on $\mathrm{Na}^{+}(\mathrm{He})_{100}$ and a triple layer structure was found [16]: the first shell was found solid-like while the third one was like liquid helium. Furthermore, our previous DMC calculations [8, 17] have found a first solvation shell of 10 helium atoms for 
$\mathrm{Li}^{+}$which is in qualitative agreement with a spatial configuration corresponding to the antiprism classical structure.

The purpose of the present work is to show that quantum stochastic calculations in small ${ }^{4} \mathrm{He}$ clusters that contain alkali cations can provide detailed evidence for both "crowding" of the solvent around the impurity (electrostriction) and the appearance of regular structures of largely equivalent adatoms (snowballs). Contrary to what has been previously done on the clusters doped with alkali metal ions we shall focus our attention only on the smaller clusters and we shall follow the evolution of their geometrical and energetic properties along the series from the dimers to the moieties containing 10-12 helium atoms. It is worth pointing out that the Potential Energy Curves (PECs) we use in the present work are different from those used in the previously mentioned studies which were instead taken from the model potentials of ref. [18]. In general our fully ab-initio PECs are slightly deeper (5\%) and our $r_{e q}$ values are by a few percent smaller. Our new PECs are described in the next Section, while Section I describes our computational methods and reports also to the features of the diatoms. In Section IV we will present the results of the triatomic clusters and Section $\mathrm{V}]$ will describe our results for the larger clusters and their snowballs structures. Finally we will summarize our present conclusions in Section VI.

\section{THE POTENTIALS}

The overall interaction in the cluster is modelled using the sum-of-potential approximation. For a cluster of $n$ helium atoms containing only one ionic impurity we therefore have:

$$
V_{\mathrm{TOT}}=\sum_{i=1}^{n} V_{\mathrm{M}^{+}+\mathrm{He}}+\sum_{i<j} V_{\mathrm{He}-\mathrm{He}}
$$

We have taken the He-He potential from Tang et al. [19] while for the $\mathrm{M}^{+}$-He potential energy curves (PECs) we have used accurate potentials calculated by us. The $\mathrm{Li}^{+}-\mathrm{He}$ potential has been already presented in refs. [8, 17] while the PECs for $\mathrm{K}^{+}-\mathrm{He}$ and $\mathrm{Na}^{+}-\mathrm{He}$ are given here for the first time. They have been calculated using the Coupled-Cluster Singles and Doubles including triple excitations non-iteratively $\operatorname{CCSD}(\mathrm{T})$ with an aug-cc-pV5Z quality basis set [20]. In the case of $\mathrm{K}^{+}$the effective core potential (ECP10MWB) [21] was used. The calculations were carried out with the MOLPRO software [22] and basis set superposition errors have been corrected via the counterpoise method [23].

The three potentials compare very well with various previous calculations and models present 


\begin{tabular}{|c|c|c|}
\hline Specie & $R_{e}$ (a.u.) & $D_{e}\left(\mathrm{~cm}^{-1} 1\right)$ \\
\hline $\mathrm{Li}^{+}-\mathrm{He}$ & $1.897^{a} 1.898^{d}$ & $649.1^{a} 646.1^{d}$ \\
\hline $\mathrm{Na}^{+}-\mathrm{He}$ & $2.324^{b} 2.323^{d}$ & $329.1^{b} 331.3^{d}$ \\
\hline $\mathrm{K}^{+}-\mathrm{He}$ & $2.825^{c} 2.839^{d}$ & $185.4^{c} 179.9^{d}$ \\
\hline
\end{tabular}

TABLE I: ${ }^{a}$ From ref. [26]; ${ }^{b}$ From ref. [24]; ${ }^{c}$ From ref. [25]; ${ }^{d}$ Present results.

in the literature, in particular we report in Table $\llbracket$ a comparison of our results with those obtained recently by Viehland et al [24, 25] for $\mathrm{K}^{+}$and $\mathrm{Na}^{+}$and by Soldán et al [26] for $\mathrm{Li}^{+}$. The data reported in the Table $\llbracket$ are $D_{e}$ and $R_{e}$. A more detailed comparison of recent and less recent data for the potentials can be found in refs. [8, 24, 25, 26].

The calculated ab-initio points have been fitted with an analytical expression which included the long range induction tail which has been chosen in order to yield at large distances the correct charge-He polarization potential $-\alpha(\mathrm{He}) / 2 r^{4}$ with $\alpha(\mathrm{He})=1.38$ a.u.. The potential functions and parameters are available on requests from the authors. More details on these potentials and on the bound states supported by them can be found in ref. [27].

When calculating the global interaction in the cluster using the sum-of-potentials approximation one neglects the contributions due to many-body effects. While these contributions are very small in neutral He clusters [28], they may be of some importance when an ionic impurity is present. In the latter case, in fact, the main contribution to the many body interaction terms comes from the fact that the He atoms near the impurity are polarized by the charge and acquire a small dipole moment. These dipoles are oriented in such a way as to contribute with a small positive interaction energy. Furthermore, in Ref. [29] for the analogous situation of the anionic dopant $\mathrm{Cl}^{-}$ in Ar clusters it has been shown that the inclusion of the leading terms of such 3-body forces (3B) does not alter substantially the energetics and the structure of the clusters. In refs. [8, 17] we have already discussed this effect in detail by carrying out a comparison of the sum-of-potentials minimum geometries with fully ab-initio ones. Our findings indicate that the addition of many-body effects does not substantially modify the geometries of the small clusters of interest in the present study. 


\section{THE MONTE CARLO CALCULATIONS}

Our numerical procedure is based on a two-step calculation. We first optimize the trial wavefunction using Variational Monte Carlo (VMC) (see for example ref. [30] for a general discussion and ref. [31] for the specific implementation). The second step is that of using the optimized wavefunction in a Diffusion Monte Carlo (DMC) algorithm [32, 33, 34] in order to obtain the energy and the geometric distributions of the cluster. We have discussed our DMC procedure more specifically in earlier work [35, 36, 37] and will therefore not be repeating it here. The form of the triel wavefunction is the familiar Jastrow function where both the He-He and the ion-He pairs have the form

$$
\log \Psi_{T}(r)=-\left(\frac{p_{5}}{r^{5}}+\frac{p_{3}}{r^{3}}+\frac{p_{2}}{r^{2}}+p_{1} r+p_{0} \log r\right)
$$

In the VMC step, the 10 parameters of the wavefunctions (He-He and ion-He) are optimized using a standard Powell algorithm [38] which minimizes a suitable estimator such as the variance of the local energy with a constant population. The population is then updated using the Metropolis sampling algorithm with a fixed wavefunction. This cycle is repeated until convergence is achieved. We noticed that in order to get a faster convergence we had to minimize in the first instance the second moment of the distribution given by

$$
\mu_{E_{R}}^{2}(H)=\sigma^{2}(H)+\left(<H>-E_{R}\right)^{2}
$$

where $E_{R}$ is a suitable reference energy near the ground state energy. In a second calculation, we then minimize directly the variance $\sigma^{2}(H)$. The parameters that we obtain are used inside a DMC program that calculates the energies and the radial distribution functions as an overall average over the walkers ensemble sampled by the mixed distribution $\Psi \cdot \Psi_{T}$ :

$$
\langle R\rangle \approx \frac{\sum_{i}^{M} w_{i} \mathbf{R}_{i}}{\sum_{i}^{M} w_{i}}
$$

In order to describe the cluster "geometry", we use different distances $\mathbf{R}_{i}$ such as the ion-He and $\mathrm{He}-\mathrm{He}$ ones or the distance of the atoms from the center of mass or from the geometric center (see ref. [39] for a more detailed definition and a further discussion on such quantities).

One important effect found in our work is the changing "size" of the alkali partner ionic core within each cluster.. The average Ion-He distances in the $n=1$ complexes range from $\sim 2 \AA$ in $\mathrm{Li}^{+}$ to $\sim 2.5 \AA$ in $\mathrm{Na}^{+}$and to $\sim 3 \AA$ in $\mathrm{K}^{+}$. As we shall see below, the size of the ion and the resulting steric effects on the solvent atom collocations are key factors in determining the features and the 
geometric structures of the first solvation shell. On the other hand, the radial delocalization of the He in the $n=1$ complex remains almost the same along the series: the average distances and their FWHM are 2.01 $\pm 0.40,2.46 \pm 0.53,3.01 \pm 0.55 \AA$ for $\mathrm{Li}^{+}, \mathrm{Na}^{+}$and $\mathrm{K}^{+}$respectively, while the minima are located at $1.91,2.30,2.93 \AA$.

\section{THE TRIATOMIC SYSTEMS}

Since we have negelected the 3B contributions to the total interaction, the trimers are the natural candidate on which we could estimate the effect of such corrections. As mentioned above, the main contribution to the $3 \mathrm{~B}$ terms in our ionic systems originates from the dipole-dipole interaction which is due to the repulsive forces between the polarized helium atoms located near the charge. As the induced dipole is inversely proportional to the distance from the ion, the size of this contribution is likely to be of some importance only for the the $\mathrm{Li}^{+}$clusters where the helium atoms are located the nearest to the ionic center. In the case of lithium, we have already shown with fully ab-initio calculations $[8,17]$ that the correct inclusion of all the many-body interactions makes the minimum structure of $\mathrm{Li}^{+} \mathrm{He}_{2}$ linear. On the contrary, when using the sum-of-potential approximation the minimum structure is triangular. Although 3B potential terms seems to play an important role in such small systems, we expect that it should become increasingly less important in larger cluster, where the crowding of the $\mathrm{He}$ atoms around the $\mathrm{Li}^{+}$core makes the repulsive short range He-He interaction the driving force in determining the geometry. Indeed, a classical minimization of a slightly larger species, the $\mathrm{Li}^{+} \mathrm{He}_{4}$ cluster, when done by either including or not including the 3B effect, produces essentially the same regular tetrahedral geometry, the only noticeable difference being the $\mathrm{R}\left(\mathrm{Li}^{+}-\mathrm{He}\right)$ distance changing from 1.89 to $1.91 \AA$ and the $\mathrm{R}(\mathrm{He}-\mathrm{He})$ distance changing from 3.09 to $3.13 \AA$ with $3 \mathrm{~B}$ forces.

We report in Table $\amalg$ the geometric parameters of the optimized structures of the $\mathrm{MHe}_{2}^{+}$clusters.. The data reported as "Quantum" are the simple averages over the final quantum distributions obtained from the DMC simulation using the sum-of-potentials approximation. The values labelled as "classical" are a simple "stick-and-ball" classical optimization that we obtained using the sum-of-potentials approximation. When comparing the classical and quantum geometrical parameters reported in Table $\amalg$ we see that our DMC results produce quantum distributions whose averages are in qualitative agreement with the classical geometries calculated with the sum-ofpotentials approximation. Obviously the quantum values imply a physical picture of He atoms 


\begin{tabular}{lccc}
\hline \hline Specie & ${ }^{7} \mathrm{Li}^{4} \mathrm{He}^{23} \mathrm{Na}^{4} \mathrm{He}^{39} \mathrm{~K}^{4} \mathrm{He}$ \\
\hline \multicolumn{4}{c}{ Quantum } \\
$<\theta>$ & 119.9 & 106.1 & 98.6 \\
$<R>(\mathrm{He}-\mathrm{He})$ & 3.38 & 3.80 & 4.38 \\
$<R>($ ion-He $)$ & 2.01 & 2.45 & 2.98 \\
\hline \multicolumn{4}{c}{ Classical } \\
$\theta$ & 102.7 & 79.2 & 63.2 \\
$\mathrm{R}(\mathrm{He}-\mathrm{He})$ & 2.96 & 2.96 & 2.96 \\
$\mathrm{R}(\mathrm{ion}-\mathrm{He})$ & 1.90 & 2.32 & 2.83 \\
\hline \hline
\end{tabular}

TABLE II: Classical geometries and quantum average values for the $\mathrm{MHe}_{2}^{+}$Clusters

which are delocalized in terms of both radial and angular density,

In figure VI we report the radial distributions of the Ion-He (upper panel) and He-He distances (lower panel).

We can see from the upper panel of Figure VI that the two He atoms are located around the ion at an average distance of 2.0, 2.5 and $3.0 \AA$ for $\mathrm{Li}^{+}, \mathrm{Na}^{+}$and $\mathrm{K}^{+}$respectively. As one can see in the lower panel of Figure $\overline{\mathrm{VI}}$, the quantum distribution of the He-He distance is much more delocalized than those of the Ion-He ones. Nevertheless, the He-He relative motion for the $\mathrm{Li}^{+} \mathrm{He}_{2}$ cluster is still confined to a relatively small region of about $1.5 \AA$. This region extends up to $2.5 \AA$ for $\mathrm{Na}^{+}$while in the case of $\mathrm{K}^{+}$the relative $\mathrm{He}-\mathrm{He}$ distance extends up to $7 \AA$ on a range of slightly less than $4.0 \AA$. This effect is obviously due to the weaker potential which exists for the $\mathrm{K}^{+}$species with respect to that of $\mathrm{Li}^{+}$, a feature which therefore induces a more marked delocalization of the relative motion of the adatoms when one goes from $\mathrm{Li}^{+}$to $\mathrm{K}^{+}$. In other words a "triangular" geometry in the three species is clearly visible although the quantum "triangle" turns out to be increasingly"floppier" when going from $\mathrm{Li}^{+} \mathrm{He}_{2}$ to the $\mathrm{K}^{+} \mathrm{He}_{2}$ moiety. 


\section{THE $\mathrm{A}^{+} \mathrm{He}_{n}$ CLUSTERS}

\section{A. The Energy Landscape}

In Figure VI (right panel) we report the evaporation energies of the present study up to sizes of $n=12$. As expected with MonteCarlo simulations our energies are affected by a statistical uncertainty which, however, is very small compared to the total energy values. This reduction is certainly due to the prior VMC optimization procedure. Such statistical errors are in fact barely visible in the right panel of Figure VI.

The evaporation energy reported by the right panel of Figure $\mathrm{VI}$ is defined as $\Delta E=$ $-\left[E\left(\mathrm{M}^{+} \mathrm{He}_{\mathrm{n}}\right)-E\left(\mathrm{M}^{+} \mathrm{He}_{\mathrm{n}-1}\right)\right]$ and represents the energy required to evaporate one He atom from the cluster. For the $\mathrm{Li}^{+}$ion we can count two jumps at $n=6$ and 8 . Each jump reflects clearly the presence of a particularly stable structure (or magic number). However, as we shall see below only the flattening at $n=10$ marks the closure of the first solvation shell (in agreement with our earlier results in [8]). An entirely different situation pertains to the $\mathrm{Na}^{+}$ion where the decrease of evaporation energy is continuous and without any marked sign of irregularities. The most important change occurs for $n=11$ which may then be a magic number. However, the first solvation shell is probably being completed between $n=11$ and $n=12$.. The behavior for $\mathrm{K}^{+}$is even smoother than that of $\mathrm{Na}^{+}$and does not present evidence of any particular magic number. The first solvation shell is likely to be filled at $n=15$ [15].

To further point out the energetic behavior of the clusters examined here, we report in the left panel of Figure VI the "normalized" evaporation energy i.e. the evaporation energy divided by the dimer energy $\left(D_{0}\right)$. The energy behavior of the three species of clusters is now independent of the strength of the particular Ion-He pair interaction: up to $n=6$, the behavior of the three clusters is thus seen to be qualitatively similar. We can say that the assembly of the cluster's structure is therefore chiefly driven by the ion-He pair potential and results in a regime with nearly additive binding energies. Geometrically we can see that the cluster grows by simply adding around the different ions the next $\mathrm{He}$ atom at a given radial distance which is similar to that identified in Table II. The $\mathrm{Li}^{+}$situation actually presents a slightly different behavior with respect to the other two species: the dimension of the central ion is so small that the crowding of the He atoms and therefore the repulsive He-He interaction in $n=4$ becomes already important in determining the structure of the cluster: the addition of one adatom destabilizes the entire cluster while for $\mathrm{Na}^{+}$ 
and $\mathrm{K}^{+}$, on the other hand, the size for which the He-He repulsive interaction becomes crucially important is $n=6$. Furthermore, for $n>6$ the specific nature of the ion is still important in determining the energetic behavior of the cluster growth: the bigger the ion, the larger the ratio $\Delta E / E$, which means that for $\mathrm{Na}^{+}$and $\mathrm{K}^{+}$one has a reduced crowding of $\mathrm{He}$ atoms around the dopant center, hence an overall smaller contribution from the He-He repulsive interactions.

\section{B. The solvation process: electrostriction effects}

It is interesting now to look at the effect that the presence of a ionic dopant has on the surrounding distribution of He atoms. As we shall show in this section, the net effect of a positive charge is that of a substantial increase of the density of He atoms surrounding the latter. This density can get to be so large that the He-He pair distribution penetrates well into the repulsive region of the He-He interaction (electrostriction effect).

In order to prove the above point, the DMC calculation shown by Figure VI report the $\mathrm{Li}^{+}$He radial density distributions together with those relative to the $\mathrm{He}-\mathrm{He}$ distance. The cluster we present in that figure have been chosen in order to show the different regimes of solvatation undergone by the central ion: up to $n=6$ we clearly see that the average radial position of the He atoms with respect to the ion (solid lines) is the same, i.e. the $6 \mathrm{He}$ atoms are part of the first shell. We can clearly see in the $\mathrm{Li}^{+} \mathrm{He}_{12}$ radial shape the appearance of a shoulder in the Ion-He radial distribution which denotes the fact that the He atom positions are not equivalent anymore. On the other hand, the first solvation shells of $\mathrm{Na}^{+}$and $\mathrm{K}^{+}$are larger than for $\mathrm{Li}^{+}$and therefore we cannot yet see this effect in their distributions. To further test this point we can look at the average distances that we get for the Ion-He pairs inside the various clusters and that are reported by Figure VI. For the $\mathrm{Li}^{+}$case, we clearly see a significant increase in the Ion-He distance after $n=6$ which is mainly due to a steric effect: on one hand the He atoms tend to assume positions which maximize the attractive branch of the He-He interaction, on the other hand they tend to reside at the shortest possible distance from the central ion, thereby minimizing the ion-He interaction: the increase in distance is a result of the energy balance between these two opposite driving forces. For the $\mathrm{Li}^{+}$ clusters we further see an increase after $n=9$ in the radial distance because the latter is now given by the average between the first and second shells. A similar behavior can also be noticed also for $\mathrm{Na}^{+}$.

The data reported in Figure VI for the He-He distributions (solid lines) show clearly the ex- 
istence of two He-He distances. For the $\mathrm{Li}^{+} \mathrm{He}_{6}$ cluster the He-He distribution is typical of an octahedral structure where we have a short distance between adjacent helium atoms and a larger one between the apical ones (see also the similar analysis carried out in [8, 40]). From the fact that the two peaks in the distribution are well separated we can say that the helium atoms are fairly "stiff" also in their relative motion. The same behavior can be found for larger structures up to $n=12$ where, however we see that the appearance of an additional peak makes the distribution more blurred: a new "shell" is being formed and the snowball is not yet completed.

If we look at the $\mathrm{He}-\mathrm{He}$ distributions for $\mathrm{Na}^{+}$and $\mathrm{K}^{+}$clusters we see clearly how the species with $n=6$ are not as yet characterized by a regular structure because those clusters are not octahedral "solids", as we shall further show below. A localization effect can be noticed, however, for the cluster containing $n=12$ atoms which has a regular structure (icosahedral) for both ions.

Putting together the information gathered from the analysis of the quantum distributions we can say that due to its small steric effect, the accretion process of the first solvation shell in $\mathrm{Li}^{+} \mathrm{He}_{n}$ is isotropic, while the same process is not isotropic for the $\mathrm{Na}^{+}$and $\mathrm{K}^{+}$ions which are slightly larger. For $\mathrm{Li}^{+}$, in fact, the structures with $n \geq 4$ are fairly compact and therefore the addition of an helium atom has the effect of increasing the radius of the solvation cage to accommodate it, while however still remaining structurally compact. The accretion of the solvation shell is therefore chiefly dominated by the strong $\mathrm{Li}^{+}-\mathrm{He}$ potential. For the other two ions, instead, the solvation process is anisotropic due to the large size of the ions which does not allow for the creation of compact structures: only for $n=9$ we see the onset of a nearly complete "spherical" solvation shell which is marked by an increased in localization of the He-He distributions (see figure VI). In order to further prove this point, we can look at the geometric distributions which locate the distances of the ion and of the He atoms from the center of mass (COM) and from the geometrical center (GC, i.e. the simple geometrical average of the positions) of the clusters (for more specific definitions, see ref. [39]). In a cluster with an isotropic distribution the COM and the GC of the entire system obviously coincide, while they have different values for an anisotropic distribution of He atoms especially when the ion is heavier than He. In Figure VI we see that for the small clusters with $n=4$ the solvation is clearly isotropic for $\mathrm{Li}^{+}$and anisotropic for $\mathrm{K}^{+}$. In other words in the specific case of $n=4$, this means that the $\mathrm{K}^{+} \mathrm{He}_{4}$ is not a tetrahedron, but (in a classical stick-and-ball model) the He atoms tend to group on one side of the ion forming an open "cup". For larger cluster this anisotropic effect obviously decreases and for the largest structures that we have analyzed here $(n=12)$ it has almost completely disappeared. 
As mentioned at the beginning of this work, "electrostriction" effects describe an increase in the density of the quantum solvent around the cationic impurity with respect to the outer region of the cluster: to show precisely this feature, we report in the upper panels of figure VI the DMC distribution of the He-He radial density superimposed on the He-He potential. One clearly sees there how, as $n$ increases (see also the distributions reported in figure VI) the He atoms get further drawn around the ion and therefore their densities show additional short-range accumulation which could be described as "tunneling" across the repulsive barrier: the destabilizing effect of entering the two body PEC repulsive region is balanced by the stabilizing contributions coming from the attractive effects of the $\mathrm{Li}^{+}-\mathrm{He}$ potential.

In contrast with the above findings, the lower panels of figure VI shows the behavior for the same cluster sizes for for $\mathrm{Na}^{+}$and $\mathrm{K}^{+}$. We clearly see there that the tunneling is somewhat reduced and therefore less significant "crowding" of the solvent adatoms occurs around the charge: the electrostriction effects, although still present, are less marked and therefore less likely to induce magic numbers in cluster growth as markedly as in the case of the $\mathrm{Li}^{+}$cation.

\section{The snowball effect}

Another important feature which characterizes the ionic dopants in He droplets is the presence of a more structured solvent environment around the ion [14, 15]: positive ions are thus surrounded by many He atoms that are strongly compressed as a result of electrostriction. The resulting ionic core is thought to be as a solid, with a diameter of several $\AA$ containing many He atoms, and is referred to as a "snowball". To investigate the possible existence and the geometric structure of such snowballs we have mapped the position of the He atoms surrounding the central ion in terms of polar angles defined via a convenient spherical coordinates system [14]. The system we use here defines a $z$ axis joining the impurity and one He atom and the $x z$ plane as the plane that contains this $z$ axis and a second He atom: all the remaining $(n-2)$ He atoms are mapped in terms of their polar angles $\theta$ and $\phi$ with respect to that reference frame. We can therefore generate an angular map of the positions of the He atoms surrounding the central charge. We focus here on the results that can be obtained for the most regular structures that we have found in our exploration of clusters with $n \leq 12$.

The $\mathrm{Li}^{+}$case has a clearly identifiable magic number for $n=6$ (although probably the first solvation shell is filled by $n=10$ ). Although $\mathrm{Na}^{+}$has no evident magic numbers, from the previous 
discussion about He-He distributions we expect a special structure for $n=12$. Also for $\mathrm{K}^{+}$we present the angular analysis for $n=12$ although the completion of the first solvation shell would probably occur at $n=15[15,40]$. In figure VI we can see the angular map of the quantum distribution and a "classical" reference map that is obtained by simply marking the angular coordinates of a regular octahedral structure obtained by a minimization of the corresponding total potential [40]. When one chooses a rigid octahedral shape, the interpretation of these maps is very simple: the $z$ axis goes through one of the vertices of the octahedron so that we can find the He atoms only at two $\theta$ values: $90^{\circ}$ and $180^{\circ}$. By fixing the $x z$ plane on a second vertex, we find the remaining three $\mathrm{He}$ atoms at the $\phi$ values $90^{\circ}, 180^{\circ}$ and $270^{\circ}$. The quantum distribution in figure VI presents three evident spots at exactly the positions of the classical rigid structure. The fourth atom, the one on the second vertex laying on the $z$ axis, has an undefined $\phi$ value, and is completely delocalized at the bottom of the quantum map where one can see a weak shading associated with its density distribution.

The striking similarities between a classical rigid structure and our quantum distribution is a consequence of the fact that the first solvation shell with such strongly interacting impurities is very much like a regular solid. Because of electrostriction, in fact, the He atoms are strongly bound and localized near the central charge.

We have encountered this "localization" effect also in the larger cluster for $\mathrm{Na}^{+}$and $\mathrm{K}^{+}$. For these ions the most regular structure is the icosahedron made of $12 \mathrm{He}$ atoms. We report in figures VI and VI the structures for $\mathrm{Na}^{+} \mathrm{He}_{12}$ and $\mathrm{K}^{+} \mathrm{He}_{12}$. Apart from the choice of the reference system, the situation is completely analogous to what we have seen for $\mathrm{Li}^{+}$: the quantum structure has a solid-like shape where the He atoms are localized (less localized for $\mathrm{K}^{+}$due to its weaker potential) at the tips of a highly regular solid icosahedra. This is the first time, to our knowledge, that such regular structures are so clearly recovered from fully quantum calculations.

\section{PRESENT CONCLUSIONS}

In the present work we have studied in detail the microsolvation process of three similar cations $\mathrm{Li}^{+}, \mathrm{Na}^{+}$and $\mathrm{K}^{+}$in a quantum solvent, ${ }^{4} \mathrm{He}$. We have employed a highly accurate quantum method which involves a combination of Variational and Diffusion Monte Carlo procedure, amply discussed in the current literature. The main approximation in the work we have presented here comes from modelling the total interaction in the clusters via a sum-of-potentials approximation 
which could lead to "unphysical" predictions for the very small moieties like $\mathrm{Li}^{+} \mathrm{He}_{2}$ : we have however shown that the inclusion of 3-Body effects in the potential seems not to alter the situation for $\mathrm{Na}^{+}$and $\mathrm{K}^{+}$and for all the clusters with $n<4$.

We have also focussed on the accretion process of the very first solvation shells around the dopant ion in order to establish geometric structures and the presence of some structural regularity in them. All the calculations have included clusters up to $n=12 \mathrm{He}$ atoms. In all the three cases the impurity is placed inside the cluster and acts as a coordination center to which the solvent atoms are bound. The radial distributions of the Ion-He distance show invariably a very high degree of localization which is mainly due to the strong Ion-He potential which dominates over the weaker $\mathrm{He}-\mathrm{He}$ interaction, at least for the smaller structures. Only for the smallest ion, $\mathrm{Li}^{+}$, we have seen a change of this behavior when passing to $n>10$ where the distribution becomes clearly made of two merged peaks, the second one representing the onset of the second solvation shell, i.e. atoms which are invariably localized at a larger distance from the ion.

The He-He distribution, on the other hand, shows a much higher degree of dispersion especially for $\mathrm{K}^{+}$. This fact can be explained by considering that the $\mathrm{K}^{+}$-He interaction is the weakest of the three PECs and that the dimension of this ion is also the biggest: the He atoms are far from the coordination center, their motions can span a much larger volume than in the case of $\mathrm{Li}^{+}$and therefore they turn out to be more delocalized. This effect somehow counteracts the electrostriction effect which consists in the increase of local He density in the proximity of the ion. Indeed we have seen how in the case of a small ion like $\mathrm{Li}^{+}$the He-He distribution clearly shows this effect by forcing the He atoms to penetrate considerably into the classically forbidden region of the HeHe pair potential. This effect is still present, although less marked, for $\mathrm{Na}^{+}$and tends to be largely absent for $\mathrm{K}^{+}$unless one considers the bigger regular structures like the one with $n=12$.

Due to this strong electrostriction effect, the $\mathrm{Li}^{+}$clusters present several magic numbers that are clearly marked by a decrease of the evaporation energy. For these clusters the solvation process remain isotropic along all the series up to $n=10$ where we have seen the filling of the first shell.

On the other hand, the magic numbers are less identifiable in the $\mathrm{Na}^{+}$clusters although we still see some structure in the dependence of the evaporation energy on cluster size. We have also computational evidence that the highly regular structure with $n=12$ marks the closure of the first solvation shell, but more calculations would be required to prove it. It is interesting to note that contrary to $\mathrm{Li}^{+}$, for $\mathrm{Na}^{+}$ions the growth of the first solvation shell is not isotropic but forms a kind of "cup" on one side of the ion as we have showed using the quantum radial distribution with 
respect to the geometric center. The situation is similar for $\mathrm{K}^{+}$where we further see no trace of magic numbers.

The shape of the quantum distributions of the angular coordinates between various atoms in the clusters allows for the presence of regular structures which show a striking similarities with their classical counterparts and a surprisingly high degree of angular localization for the solvent atoms: one clearly sees very ordered structures typical of solid-like geometries. This quantum analysis confirms the qualitative validity of a the snowball model although points out that the snowball structures, as well as their spatial extension, strongly depend on the chemical nature of the dopant ion.

\section{Acknowledgments}

Financial support from the EU Network COLMOL HPRN-CT-2002-00290, from the Scientific Committee of the University of Rome, from the Ministry of University and Research (MIUR) Nationa Projects (PRIN) and from the CASPUR Supercomputing Center are all gratefully acknowledged. We also thank the Agnelli Foundation for financing visits between Rome and Koć universities during the development of the present research.

[1] J. P. Toennies and A. F. Vilesov, Ann. Rev. Phys. Chem. 49, 1 (1998).

[2] J. P. Toennies, A. F. Vilesov, and K. B. Whaley, Phys. Today 54, 31 (2001).

[3] F. Stienkemeier and A. F. Vilesov, J. Chem. Phys. 115, 10119 (2001).

[4] J. P. Toennies and A. F. Vilesov, Angew. Chem. Int. Ed. 43, 2622 (2004).

[5] F. Stienkemeier and K. K. Lehmann, J. Phys. B 39, R127 (2006).

[6] M. Barranco, G. R., S. Hernández, R. Mayol, J. Navarro, and M. Pi, J. Low Temp. Phys. 142, 1 (2006).

[7] E. Bodo, F. Sebastianelli, F. A. Gianturco, E. Yurtsever, and M. Yurtsever, J. Chem. Phys. 120, 9160 (2004).

[8] C. D. Paola, F. Sebastianelli, E. Bodo, I. Baccarelli, F. A. Gianturco, and M. Yurtsever, J. Chem. Theory and Comput. 1, 1045 (2005).

[9] R. Guardiola, O. Kornilov, J. Navarro, and J. P. Toennies, J. Chem. Phys. 124, 084307 (2006).

[10] R. Melzer and J. G. Zabolitzky, J. Phys. A 17, L565 (1984). 
[11] K. R. Atkins, Phys. Rev. 116, 1339 (1959).

[12] N. Takahashi, T. Shigematsu, S. Shimizu, K. Horie, Y. Hirayama, H. Izumi, and T. Shimoda, Physica B 89, 284 (2000).

[13] T. MacFarland, S. A. Vitiello, L. Reatto, G. V. Chester, and M. H. Kalos, Phys. Rev. B 50, 13577 (1994).

[14] D. E. Galli, M. Buzzacchi, and L. Reatto, J. Chem. Phys. 115, 10239 (2001).

[15] M. Rossi, M. Verona, D. E. Galli, and L. Reatto, Phys. Rev. B 69, 212510 (2004).

[16] A. Nakayama and K. Yamashita, J. Chem. Phys. 112, 10966 (2000).

[17] F. Sebastianelli, I. Baccarelli, E. Bodo, C. di Paola, F. A. Gianturco, and M. Yurtsever, Comp. Mater. Sci. 35, 261 (2005).

[18] A. D. Koutselos, E. A. Mason, and L. A. Viehland, J. Chem. Phys. 93, 7125 (1990).

[19] K. T. Tang, J. P. Toennies, and C. L. Yiu, Phys. Rev. Lett. 74, 1546 (1995).

[20] E. P. F. Lee and T. G. Wright, Chem. Phys. Lett. 363, 139 (2002).

[21] T. Leininger, A. Nicklass, W. Kchle, H. Stoll, M. Dolg, and A. Bergner, Chem. Phys. Lett. 255, 274 (1996).

[22] H.-J. Werner, P. J. Knowles, R. Lindh, F. R. Manby, M. Schütz, et al., Molpro, version 2006.1, a package of ab initio programs (2006), see http://www.molpro.net

[23] S. F. Boys and F. Bernardi, Mol. Phys. 19, 553 (1970).

[24] L. A. Viehland, J. Lozeille, P. Soldan, E. P. F. Lee, and T. G. Wright, J. Chem. Phys. 119, 3729 (2003).

[25] L. A. Viehland, J. Lozeille, P. Soldan, E. P. F. Lee, and T. G. Wright, J. Chem. Phys. 121, 341 (2004).

[26] P. Soldán, E. P. Lee, J. Lozeille, J. N. Murrel, and T. G. Wright, Chem. Phys. Lett. 343, 429 (2001).

[27] F. Marinetti, E. Coccia, E. Bodo, F. A. Gianturco, E. Yurtsever, M. Yurtsever, and E. Yildirim, Theor. Chem. Acc. p. accepted (2007).

[28] I. Baccarelli, F. Giantuco, T. Gonzalez-Lezana, G. Delgado-Barrio, S. Miret-Arts, and P. Villarreal, J. Chem. Phys. 122, 144319 (2005).

[29] T. Lenzer, I. Yourshaw, M. R. Furlanetto, N. L. Pivonka, and D. M. Neumark, J. Chem. Phys. 115, 3578 (2001).

[30] D. Bressanini, G. Morosi, and M. Mella, J. Chem. Phys. 116, 5345 (2002).

[31] A. Mushinski and M. Nightingale, J. Chem. Phys. 101, 8831 (1994).

[32] J. B. Anderson, Int. Rev. Phys. Chem. 14, 85 (1995).

[33] D. M. Ceperly and B. Alder, Science 231, 555 (1986). 
[34] A. Viel, M. V. Patel, P. Niyaz, and K. B. Whaley, Comp. Phys. Comm. 145, 24 (2002).

[35] F. Paesani and F. A. Gianturco, J. Chem. Phys. 116, 10170 (2002).

[36] F. Paesani and F. A. Gianturco, J. Chem. Phys. 117, 709 (2002).

[37] F. Paesani, A. Viel, F. A. Gianturco, and K. B. Whaley, Phys. Rev. Lett. 90, 073401 (2003).

[38] W. Press, B. Flannery, S. Teukolsky, and W. Vetterling, Numerical Recipes (Cambridge University Press, 1986).

[39] C. di Paola and F. A. Gianturco, Eur. Phys. J. D 35, 513 (2005).

[40] E. Bodo, E. Yurtsever, M. Yurtsever, and F. A. Gianturco, J. Chem. Phys. 124, 074320 (2006). 


\section{caption to figures}

- Fig 1: DMC radial distribution of the Ion-He and He-He distances in the $n=2$ cluster

- Fig 2: Right: Evaporation energy $\Delta E=-\left[E\left(\mathrm{M}^{+} \mathrm{He}_{\mathrm{n}}\right)-E\left(\mathrm{M}^{+} \mathrm{He}_{\mathrm{n}-1}\right)\right]$. Left: Dimensionless $\Delta E / E$ evaporation function

- Fig 3: Radial $\mathrm{A}^{+}-\mathrm{He}$ and He-He DMC distribution for $\mathrm{A}^{+} \mathrm{He}_{n}$. Distances in $\AA$. Ditributions in $\AA^{-1}$

- Fig 4: Average distance between the ion and the He atoms in the three series of clusters as a function of size. Distances are in $\AA$.

- Fig 5: Radial distribution of the distance from the center of mass and the geometrical center of the cluster. Left panel: $\mathrm{Li}^{+}$, right one: $\mathrm{K}^{+}$. A schematic view of the cluster geometry is also depicted: in the case of $\mathrm{K}^{+}$the GC is indicated by a cross.

- Fig 6: Radial He-He DMC distribution for $\mathrm{A}^{+} \mathrm{He}_{n}$ superimposed to the He-He potential. Distances in $\AA$ and energy in $\mathrm{cm}^{-1}$.

- Fig 7: Angular correlation maps for $\mathrm{Li}^{+} \mathrm{He}_{6} . \theta$ is on the $y$ axis and $\phi$ on the $x$ one. The white dots are the position of the corresponding localized minimum structure which is also reported in the small inset.

- Fig 8: As in figure 7 but for $\mathrm{Na}^{+} \mathrm{He}_{12}$.

- Fig 9: As in figure 7 but for $\mathrm{K}^{+} \mathrm{He}_{12}$. 


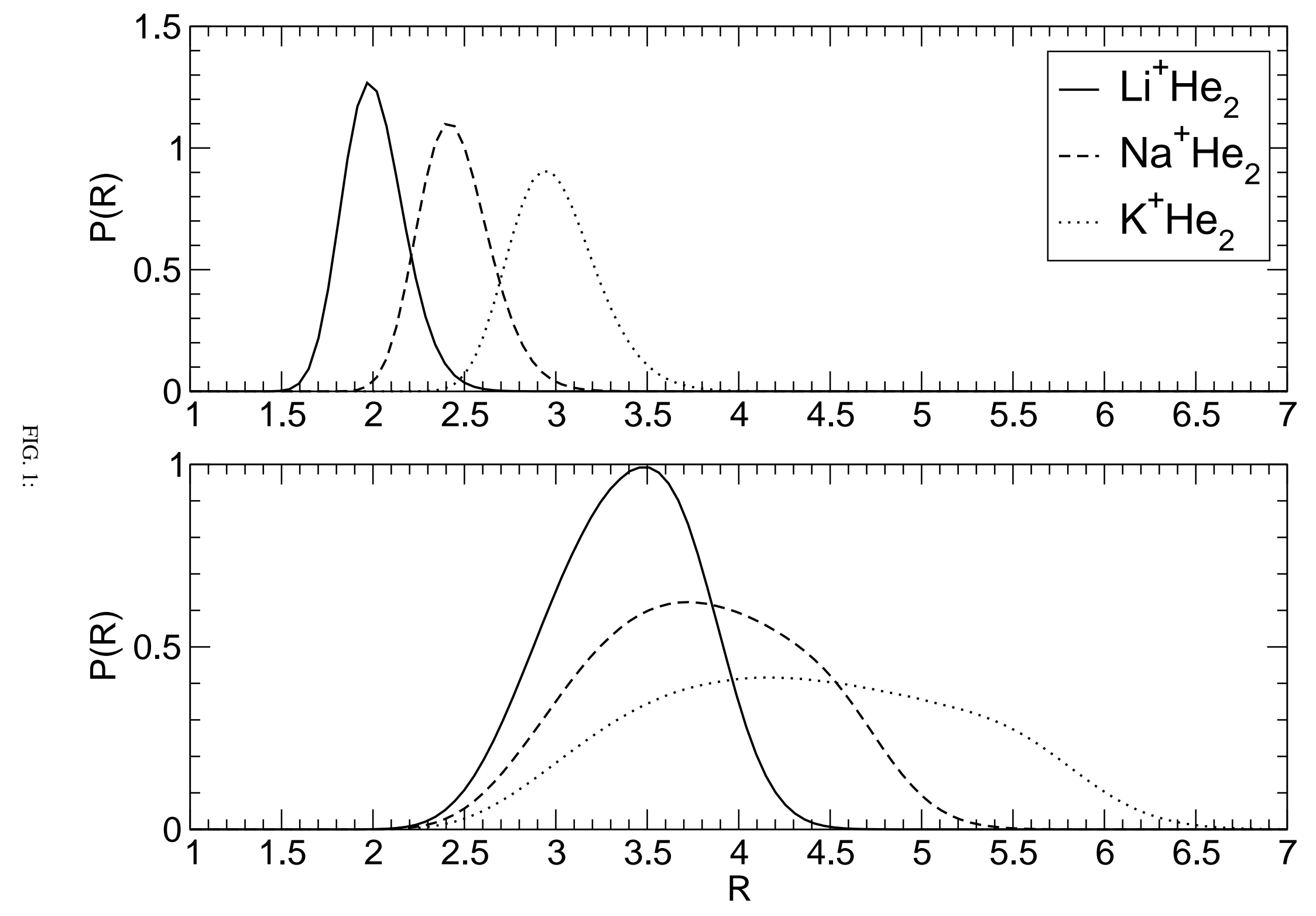




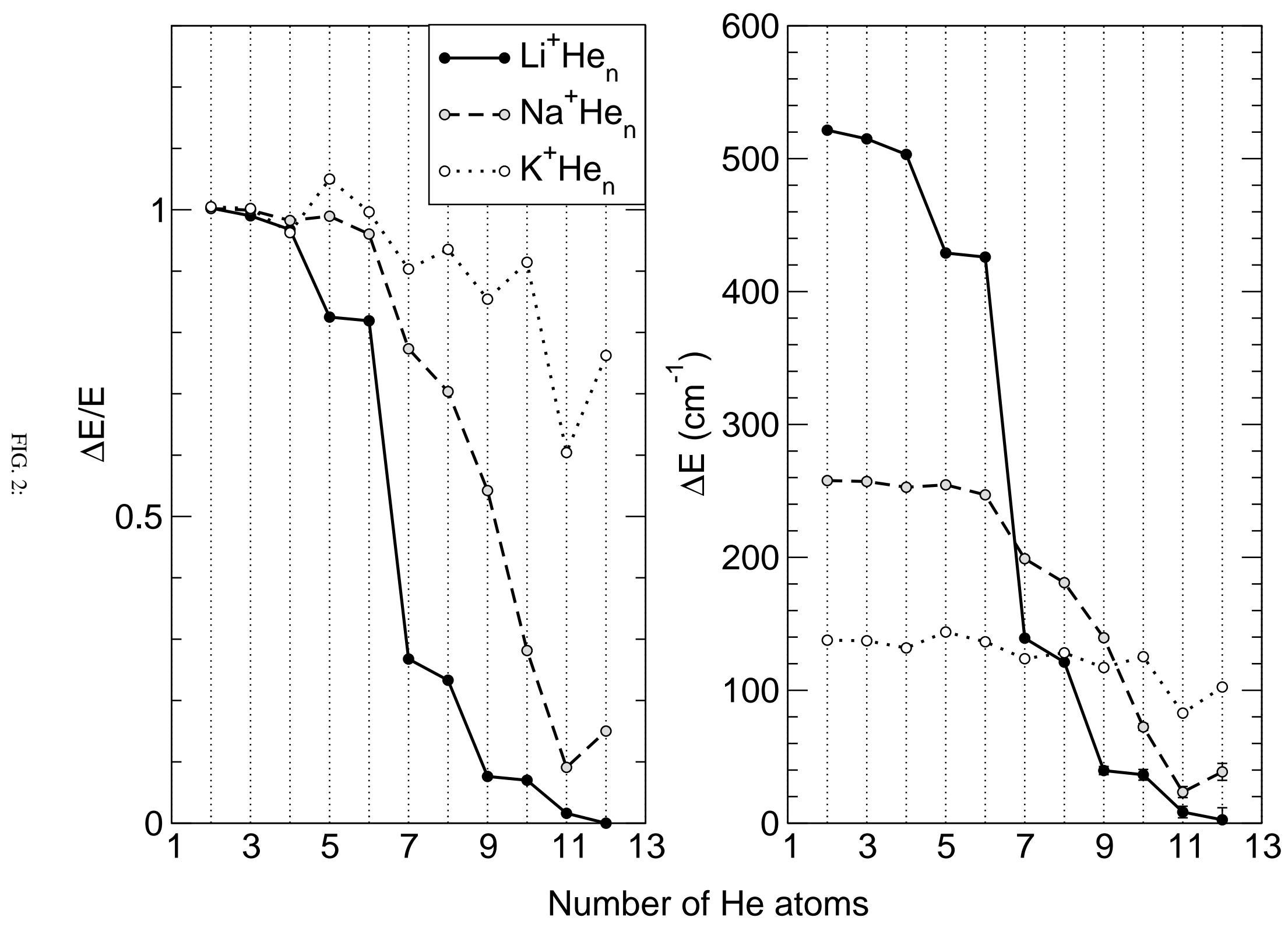



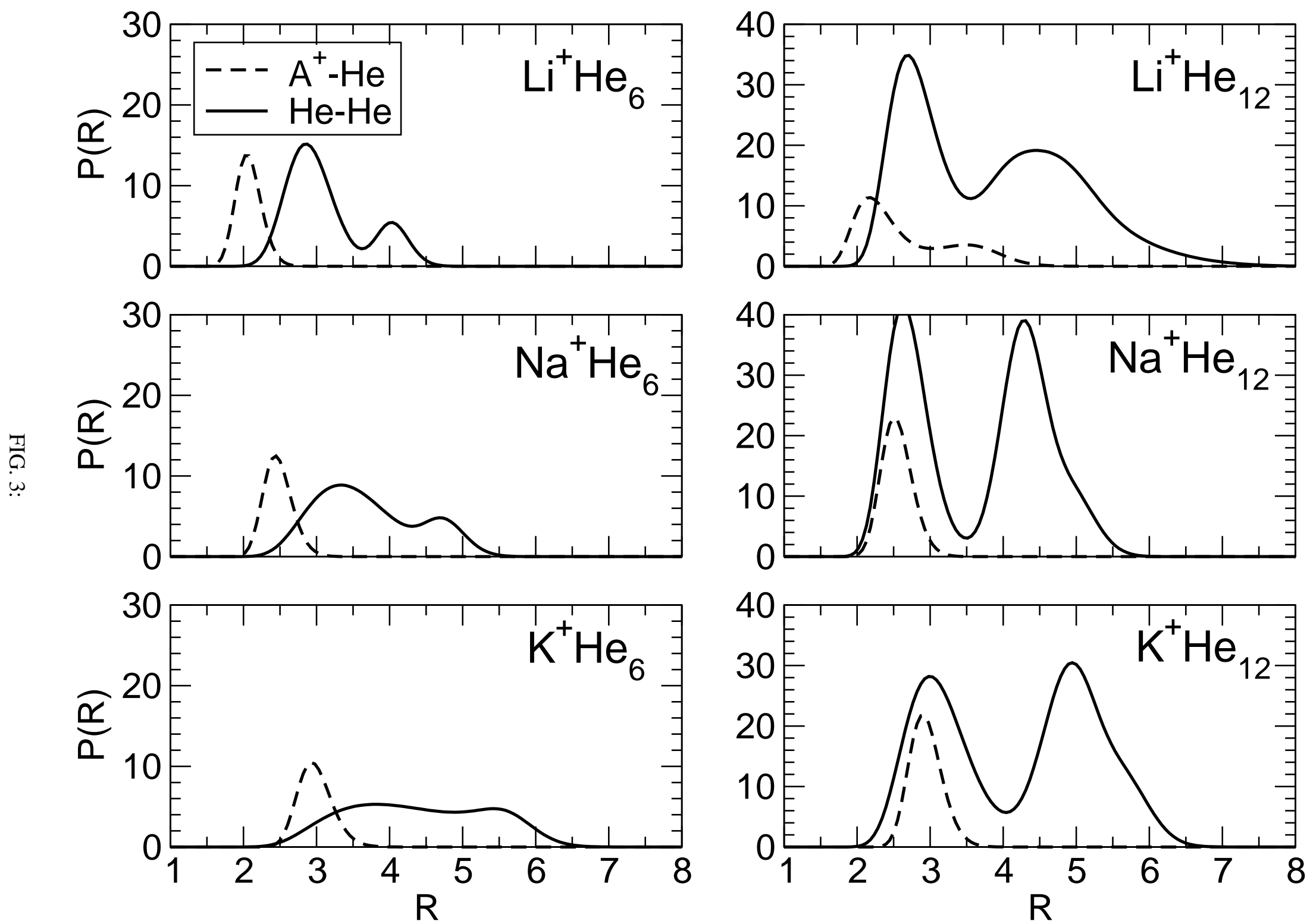


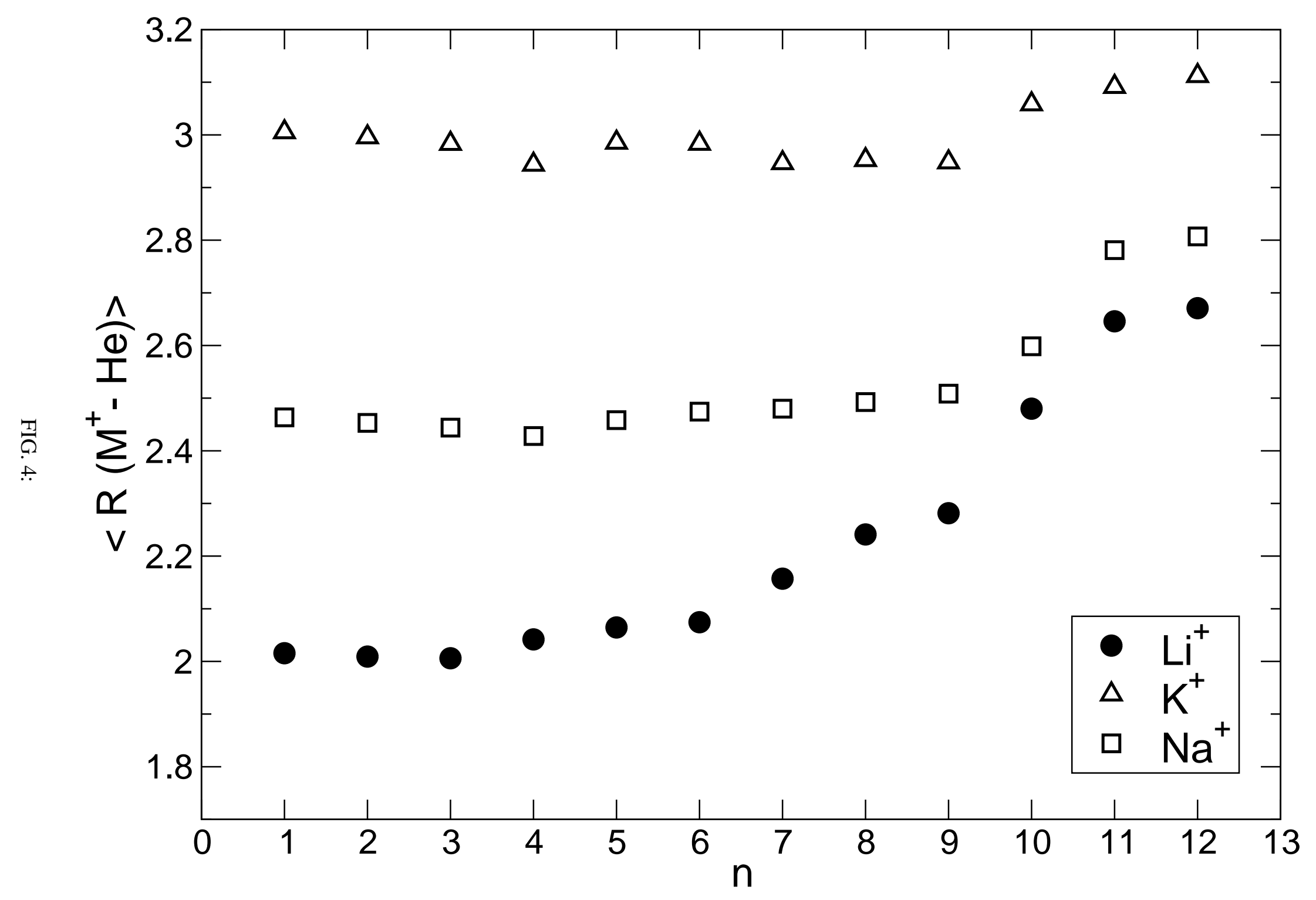



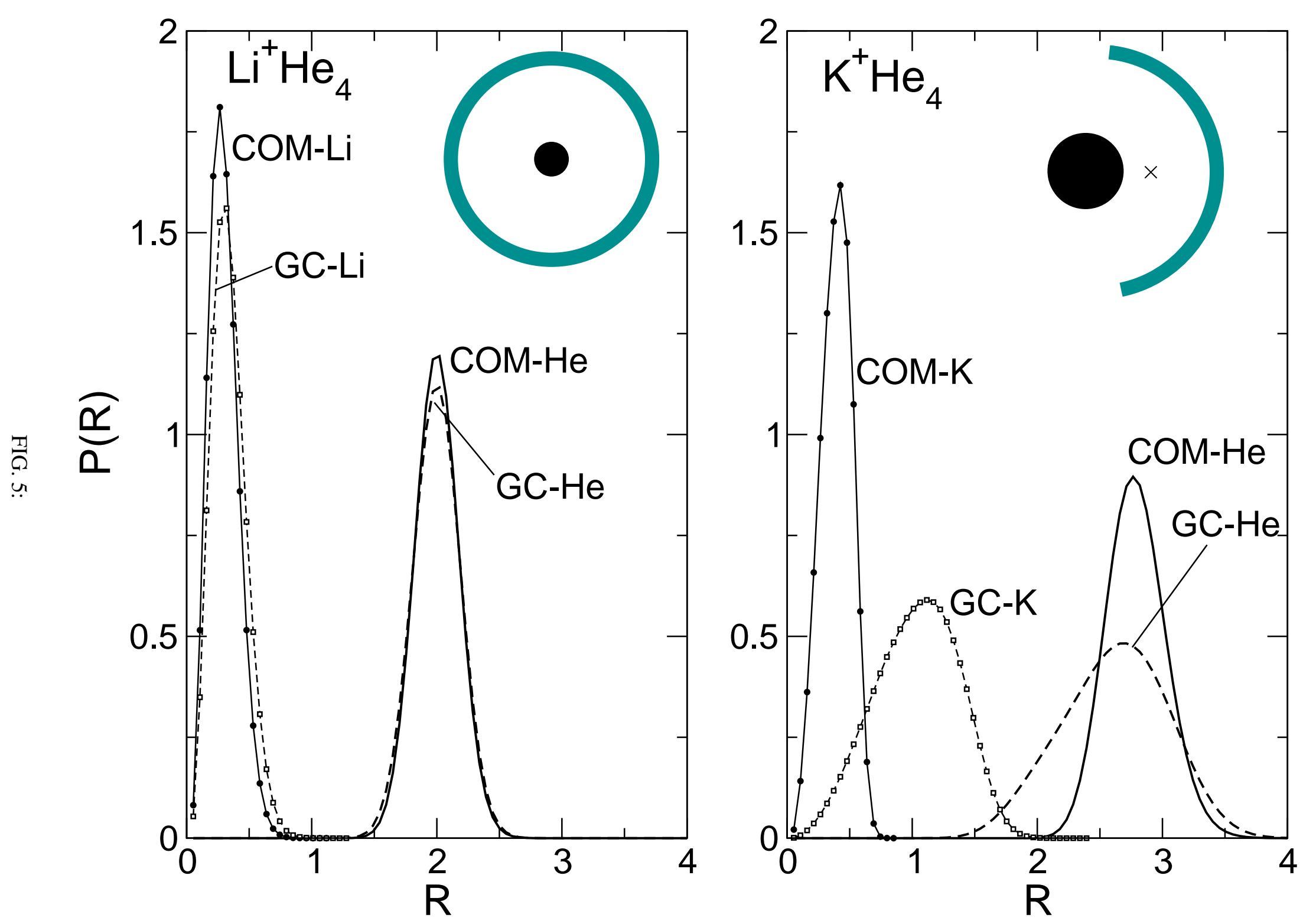

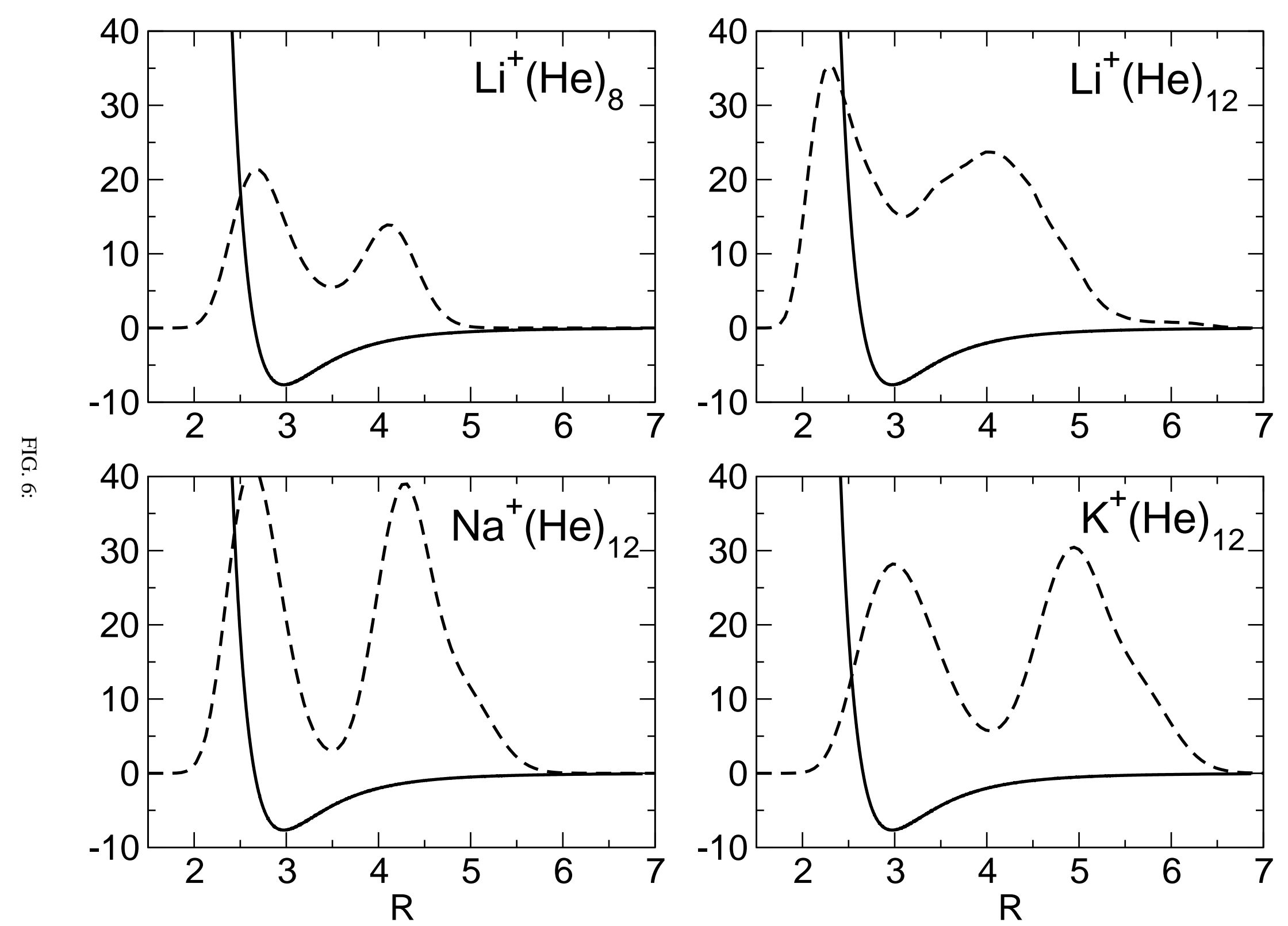


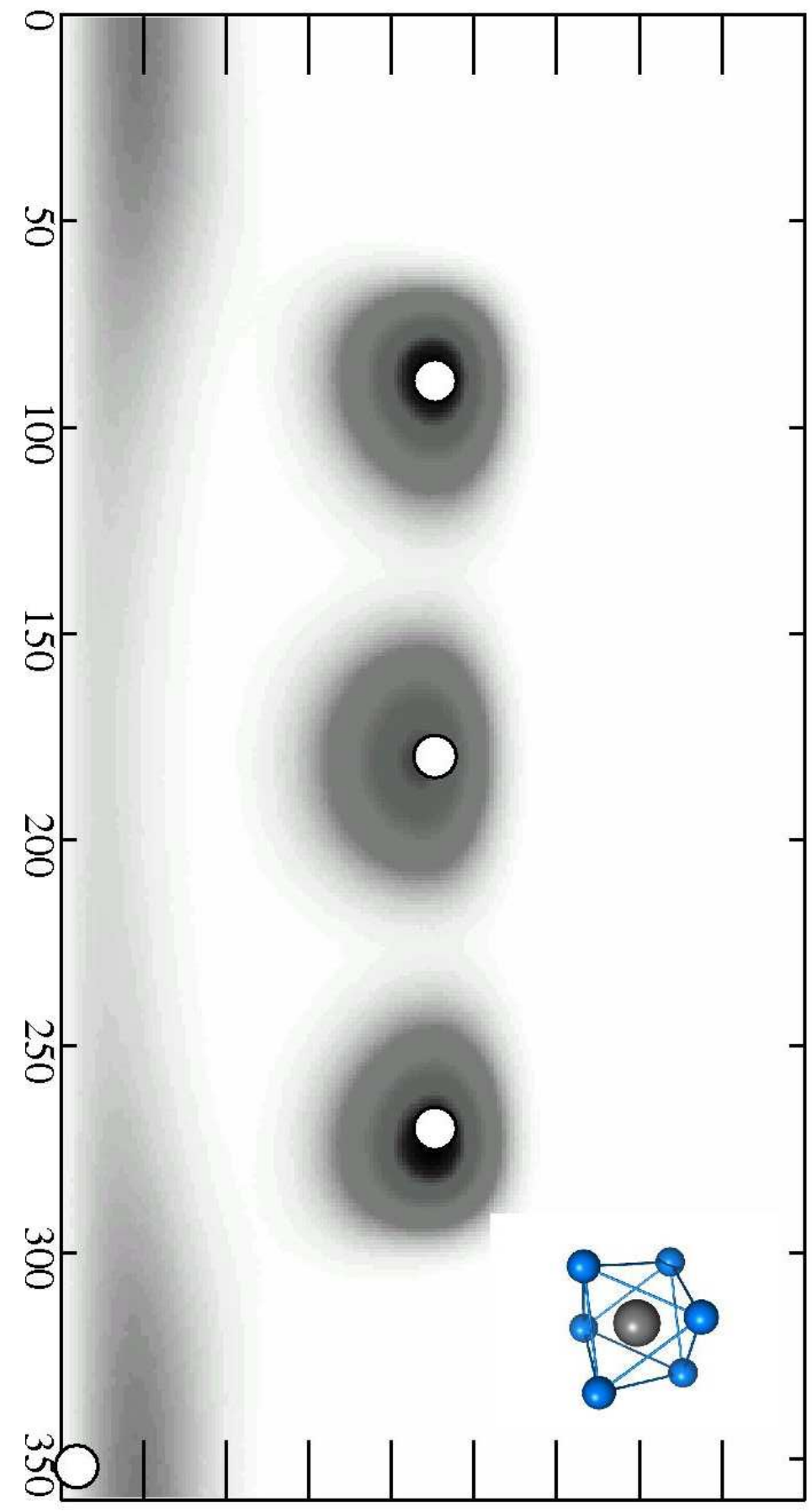

क

FIG. 7: 


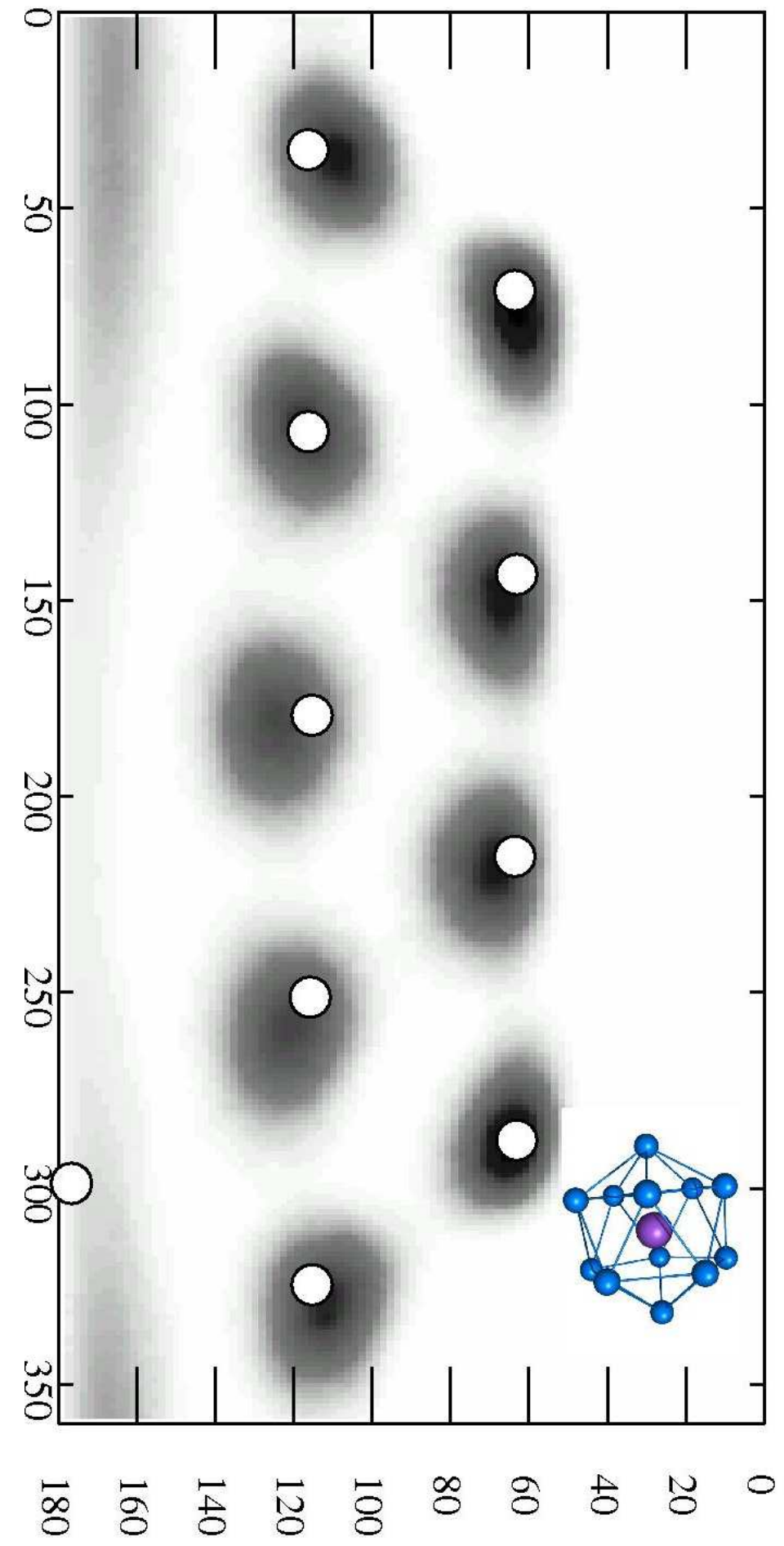

FIG. 8: 


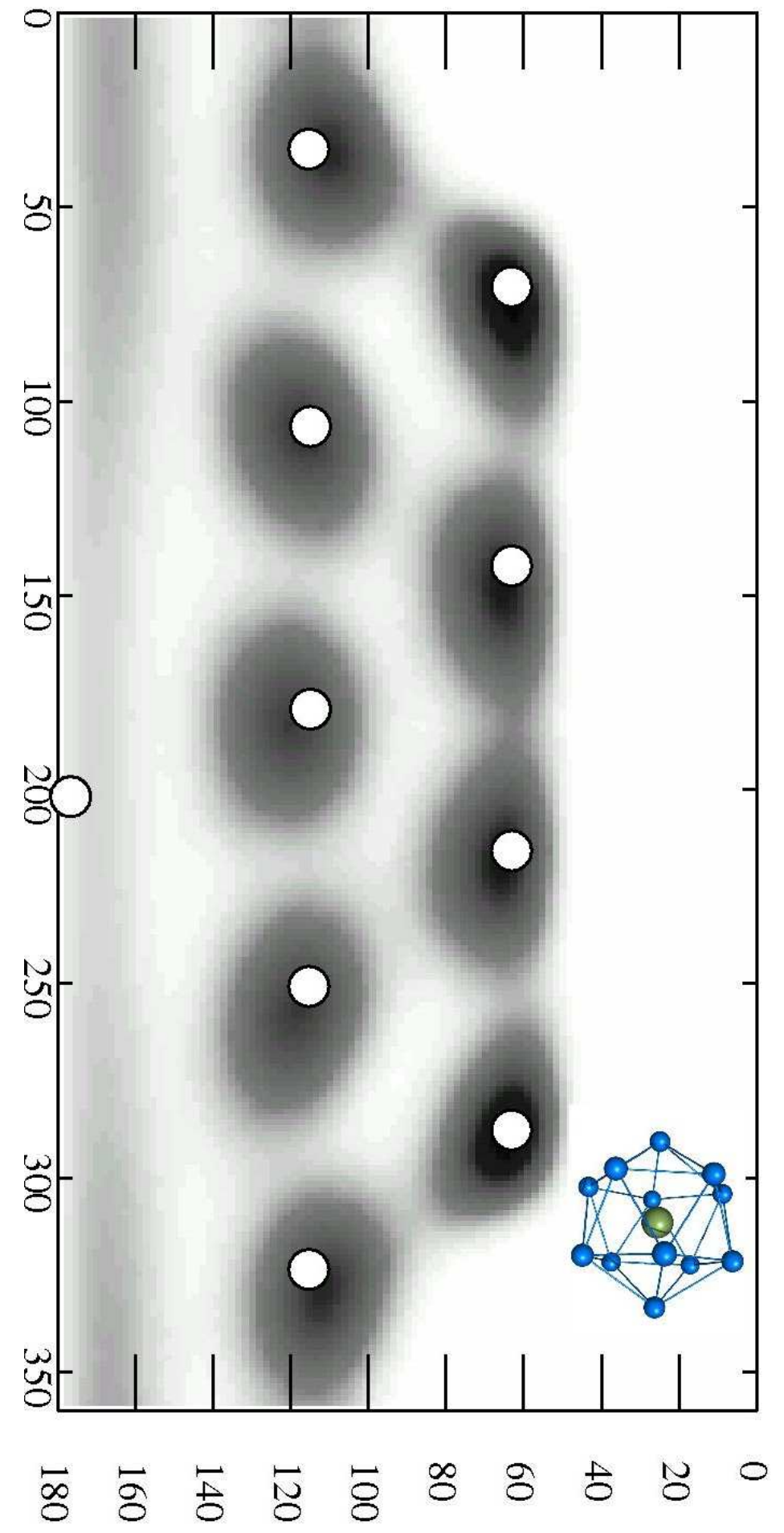

FIG. 9: 